\title{
Dependence of the electronic and crystal structure of a functionalized graphene on the concentration of chemically adsorbed fluorine
}

\author{
M. E. Belenkov, V. M. Chernov \\ Chelyabinsk State University, Br. Kashirinykh, 129, Chelyabinsk, 454001, Russia \\ me.belenkov@gmail.com
}

PACS 61.50.Ah, 81.05.ue

DOI 10.17586/2220-8054-2020-11-6-685-689

\begin{abstract}
In this paper, we study the change in the fluorine-functionalized graphene layers depending on the fluorine concentration. Ab initio calculations were performed using the density functional theory method in the generalized gradient approximation. It was established that the metallic properties of the graphene layer become semiconducting after functionalization even at low concentrations of chemically adsorbed fluorine $\sim 10$ at. $\%$. The band gap increases from 0.11 to $3.09 \mathrm{eV}$ with an increase of the amount of adsorbed fluorine.
\end{abstract}

Keywords: graphene, fluorographene, ab initio calculations, chemical adsorption, crystal structure, electronic properties.

Received: 10 October 2020

Revised: 27 October 2020

\section{Introduction}

Graphene can be functionalized by chemical adsorption of non-carbon atoms on its surface, as a result of which its properties change from metallic to semiconducting [1,2]. Various non-carbon atoms and molecular groups are chemically well adsorbed on the graphene surface [3,4]. Fluorographene is the most promising for practical applications in electronics $[5,6]$. The thermal stability of graphene functionalized by fluorine is higher than that of graphene functionalized with hydrogen, oxygen or chlorine [7-9]. The electronic properties of graphene functionalized by fluorine can be influenced by the order of addition of adsorbed fluorine atoms [10-13], as well as a number of other factors. So, in works $[14,15]$ it was found that the electronic structure of graphene changes during the chemical adsorption of individual fluorine atoms. A detailed study of the dependence of the graphene electronic properties on the concentration of chemically adsorbed fluorine was not in the scope of those prior works. Therefore, in this paper, such studies were carried out.

\subsection{Methods}

$\mathrm{Ab}$ initio modeling of fluorine-functionalized graphene compounds was performed using the generalized gradient approximation for density functional theory (DFT-GGA) [16]. The calculations were performed using the QUANTUM Espresso software package [17]. An extended hexagonal unit cell containing 18 carbon atoms was chosen as the main unit cell of the initial graphene layer. During chemical adsorption, fluorine atoms were randomly attached to carbon atoms in a unit cell, and then the optimized crystal structure, band structure, and density of electronic states were calculated. We have considered the compounds obtained by the addition of $0,2,4,6,8,10,12,14,16$ and 18 fluorine atoms to the extended unit cell of the graphene layer, which corresponds to the fluorine concentration in the compounds $0,10,18.2,25,30.8,35.7,40,43.8,47.1,50$ at.\%. We have considered only those structures in which half of the fluorine atoms were attached on one side of the layer, and the other half of the atoms on the other. The calculations were performed for fluorine functionalized graphene layers, which were packed in stacks. The distance between the layers in the stacks was large $(12 \AA)$; therefore, adjacent layers did not affect the structure of each other. The calculations used a set of $k$-points $12 \times 12 \times 12$. The wave functions were decomposed using a truncated basis set of plane waves. The cutoff energy for the plane wave basis was 70 Rydberg.

\subsection{Results}

As a result of DFT-GGA calculations, the structure of fluorine-functionalized graphene layers was found, which corresponds to the minimum total energy. Images for four calculated layers corresponding to fluorine concentrations in the structure of 10, 25, 35.7, and 43.8 at.\% are shown in Fig. 1. At a low fluorine concentration, the structure of the graphene layer remains flat, and deformation of the structure is observed only around the attached fluorine atom. With increasing concentration, the layer gradually becomes corrugated, as a result the carbon atoms are displaced relative to the initial plane of the layer in the direction of the attached fluorine atoms. 


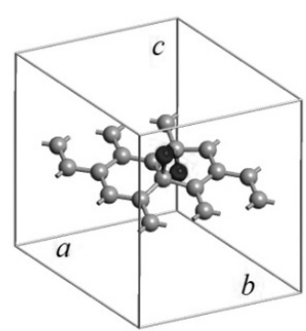

(a)
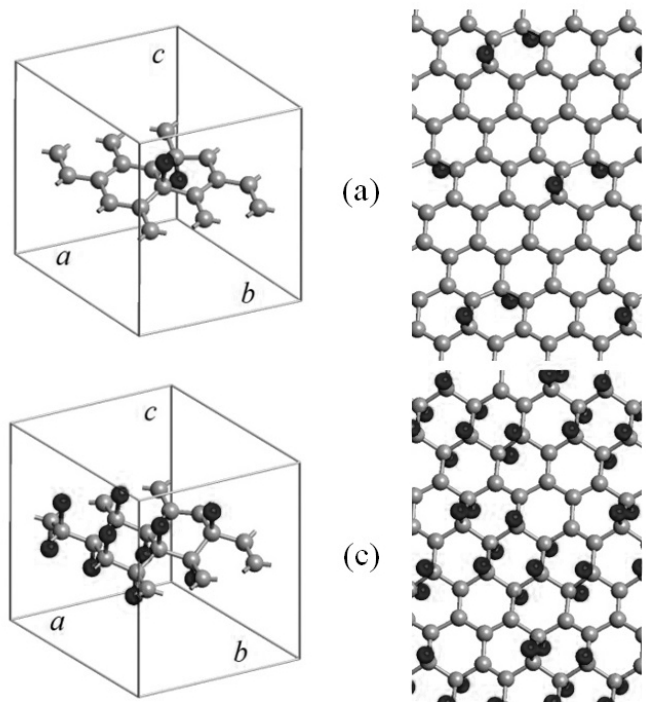

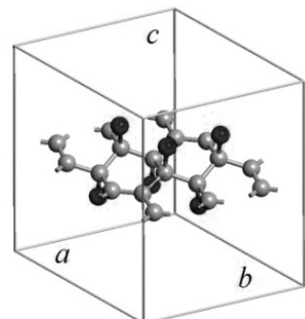

(b)

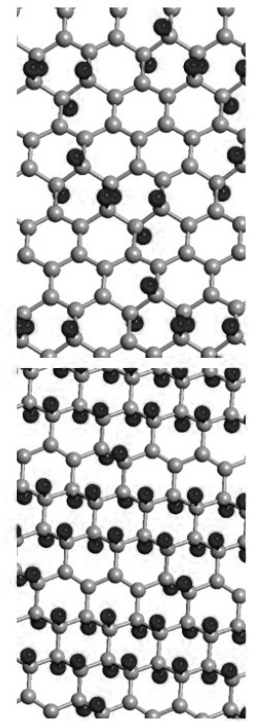

FIG. 1. The unit cells and the structure of graphene layers with different amount of chemically adsorbed fluorine atoms per unit cell: (a) 2 fluorine atoms; (b) 6 fluorine atoms; (c) 10 fluorine atoms; (d) 14 fluorine atoms. The unit cells contain 18 carbon atoms

The calculated values of the lattice translation vector lengths of graphene layers are given in Table 1. The minimum value of the translation vector length $(7.4608 \AA)$ is observed for the initial graphene layer. The maximum vector length $(7.8050 \AA)$ corresponds to a completely fluorinated graphene layer in which a fluorine atom is attached to each carbon atom. An increase in the crystal lattice parameter with an increase in fluorine concentration occurs despite the fact that initially flat layers are corrugated and if the interatomic carbon-carbon bonds remained constant, then an inverse relationship should have been observed. The observed increase in the parameter is associated with the fact that the order of carbon-carbon bonds changes and their average length increases. The change in the unit cell parameter of the initial graphene in comparison with fully fluorinated graphene was observed earlier in the article [18]. According to the data of this article, the experimentally measured lattice parameter of the graphene layer is $0.246 \mathrm{~nm}$, and for fluorographene it is $0.257 \mathrm{~nm}$. In our calculations, we used an extended unit cell with a translation vector 3 times larger than that of a primitive unit cell. Therefore, if the parameters of the unit cells we found are divided by 3 , that is, reduced to a primitive cell, then the translation vector for graphene will be $0.249 \mathrm{~nm}$, and for fluorographene, $0.260 \mathrm{~nm}$. The calculated data obtained by us are in good agreement with the experimentally measured values. The dependence of the change in the lattice parameter on the concentration of adsorbed fluorine found in our work can find practical application for determining the concentration of fluorine from the experimentally measured values of the lattice parameter.

With an increase in the fluorine concentration in the compounds, the C-F bond length also changes from $1.634 \AA$ in the graphene layer with a fluorine concentration of 10 at.\% to $1.439 \AA$ in the fluorographene layer, where the fluorine concentration is 50 at.\%. Apparently, the reason for this is weaker chemical bond between fluorine and carbon atoms in graphene layers with a low fluorine concentration as compared to $\mathrm{C}-\mathrm{F}$ bonds in the fluorographene layer. Calculations performed for carbon tetrafluoride molecules with different interatomic bond lengths have shown that the weakening of the C-F bond energy corresponding to the observed change in the bond length for fluorinated graphene layers can reach $11 \%$.

The calculated values of the total energy Etotal per unit cell are presented in Table 1. This energy decreases with an increase of the number of fluorine atoms attached to the graphene layer. In this case, the energy of interatomic chemical bonds Ebind also decreases. The bond energy was calculated as the difference between the total energy and the energy of the same number of isolated carbon and fluorine atoms as in the unit cell of the corresponding layer. The decrease in bond energy with an increase in the number of atoms first and foremost is due to an increase in the number of bonds. Calculation of the energy of carbon-carbon EC-C bonds showed that the energy of this bond gradually increases with increasing fluorine concentration. Indeed, in the initial graphene layer, the binding energy between a pair of neighboring carbon atoms was $-6.881 \mathrm{eV}$, while in the fluorographene layer this energy is $-4.926 \mathrm{eV}$. The energies of carbon-carbon bonds $(-6.88 \div-4.93 \mathrm{eV})$ found as a result of calculations correlate well with the experimentally established values of the energies of single and double carbon-carbon bonds $(-7.05$, 
TABLE 1. Structural parameters, energy characteristics, and electronic properties of a graphene layer functionalized with fluorine with different numbers of chemically adsorbed fluorine atoms $\left(N_{F}\right.$ - the number of fluorine atoms adsorbed into an expanded unit cell of the graphene layer, which contains 18 carbon atoms; $a$ - the lattice translation vector; $R_{C-F}$ - the average bond length between a fluorine and carbon atom; $E_{\text {total }}$ - the total bond energy per unit cell; $E_{\text {bind }}$ - the energy of all bonds in a unit cell; $E_{\mathrm{C}-\mathrm{C}}-$ the average energy of one carbon-carbon bond; $E_{F}-$ the Fermi energy; $\Delta-$ the band gap)

\begin{tabular}{cccccccc}
\hline$N_{F}$, at. & $a, \AA$ & $R_{C-F}, \AA$ & $E_{\text {total }}, \mathrm{eV}$ & $E_{\text {bind }}, \mathrm{eV}$ & $E_{C-C}, \mathrm{eV}$ & $E_{F}, \mathrm{eV}$ & $\Delta, \mathrm{eV}$ \\
\hline 0 & 7.4608 & - & -2831.65 & -185.78 & -6.881 & -4.054 & 0 \\
2 & 7.4609 & 1.634 & -4117.22 & -192.70 & -6.623 & -4.721 & 0.114 \\
4 & 7.4672 & 1.506 & -5403.59 & -200.42 & -6.396 & -5.312 & 1.573 \\
6 & 7.4978 & 1.485 & -6689.77 & -207.95 & -6.161 & -5.647 & 1.092 \\
8 & 7.5381 & 1.473 & -7976.20 & -215.73 & -5.936 & -5.965 & 1.785 \\
10 & 7.5708 & 1.471 & -9262.02 & -222.91 & -5.688 & -6.413 & 1.338 \\
12 & 7.6235 & 1.453 & -10549.3 & -231.54 & -5.494 & -6.487 & 1.779 \\
14 & 7.6948 & 1.458 & -11836.8 & -240.39 & -5.308 & -6.402 & 2.445 \\
16 & 7.7698 & 1.442 & -13123.9 & -248.84 & -5.108 & -6.201 & 2.909 \\
18 & 7.8050 & 1.439 & -14411.5 & -257.80 & -4.926 & -6.053 & 3.094 \\
\hline
\end{tabular}

$-3.84 \mathrm{eV}$ ) [19], which indicates the correctness of the calculations. During fluorination, the energy of C-C bonds changes by about one third of the energy of the initial graphene. This is due to the fact that in the initial graphene layer the carbon bond order is 1.33 , and in the fluorographene layer the bond order becomes 1 . Therefore, the bond energy changes proportionally. Such a change in the binding energy should lead to a decrease in the mechanical properties of fluorinated graphene in comparison with the properties of the initial graphene layer.

DFT-GGA calculations of the band structure and the density of electronic states showed that they change significantly with an increase in the concentration of adsorbed graphene. Figs. 2 and 3 show examples of plots for some layers in which the fluorine concentration sequentially increases from 10, 25, 35.7, and 43.8 at.\%. At the Fermi energy level, the band gap changes are the most clear when the concentration of fluorine changes. If there is no band gap in the initial graphene layer and there is a contact between the valence and conduction bands at the Fermi energy level, then a band gap appears during fluorine adsorption. At a fluorine concentration of 10 at. $\%$, the band gap remains small at $0.114 \mathrm{eV}$ (Fig. 2a, Fig. 2b, Table 1). With an increase of concentration, an increase of the band gap is observed (Fig. 4). This growth is not monotonous: in the concentration range $10-15$ at.\%, a jump in the band gap to $\sim 1.5 \mathrm{eV}$ is observed, and then the band width gradually increases with increasing fluorine concentration, reaching $3.094 \mathrm{eV}$ for a completely fluorinated graphene layer. The band gap $(0.114 \mathrm{eV})$ found by us for the low fluorine concentration correlates well with the results given in [11] $(0.118-0.400 \mathrm{eV})$ for a similar concentration. As a result of calculations of the electronic structure of the initial graphene layers, it was found that the band gap at the Fermi energy level for this layer is 0 . This is in good agreement with the data of theoretical calculations performed earlier by other authors [20], as well as the results of experimental research [21]. The calculated value of the band gap in fully fluorinated graphene is $3.094 \mathrm{eV}$, which is in good agreement with the previously found theoretical values $(2.7-3.5 \mathrm{eV}$ [22], 3.07 eV [23]) and experimental values ( $3 \mathrm{eV}[6])$. Therefore, the bandgaps calculated by us for partially fluorinated graphene layers should also be correct.

\subsection{Conclusion}

Thus, in this work, we have performed calculations of changes in the structure and electronic characteristics of graphene layers with different concentrations of adsorbed fluorine atoms. It was found that an increase in the fluorine concentration leads to an increase in the unit cell parameter of the layer (from 7.4608 to $7.8050 \AA$ ), a decrease in the energy of the carbon-carbon bond (from -6.881 to $-4.926 \mathrm{eV}$ ), and an increase in the band gap (0 to $3.094 \mathrm{eV})$. The results obtained in this work can be used to synthesize new graphene-based materials for micro- and nanoelectronics. 

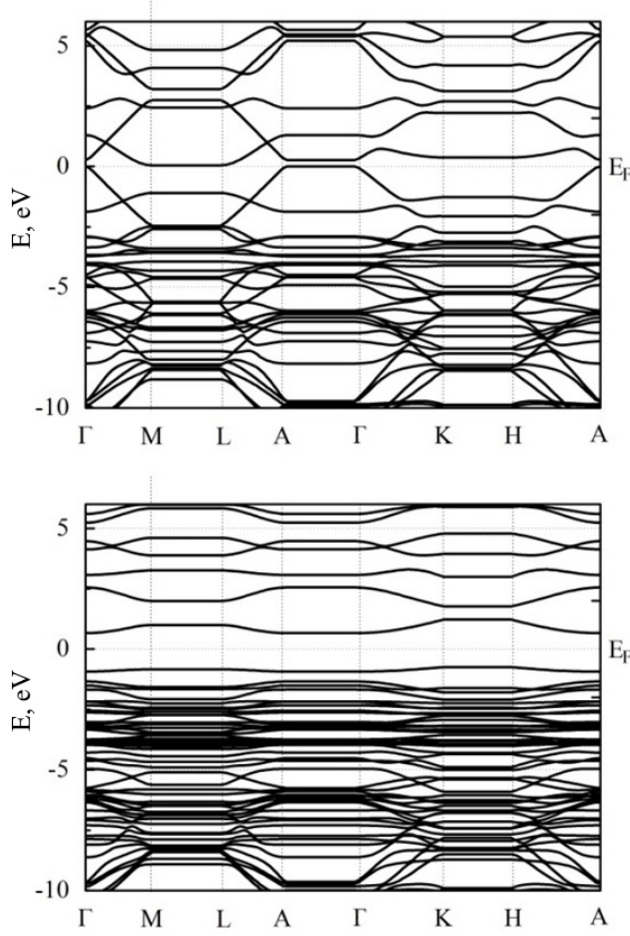

(a)

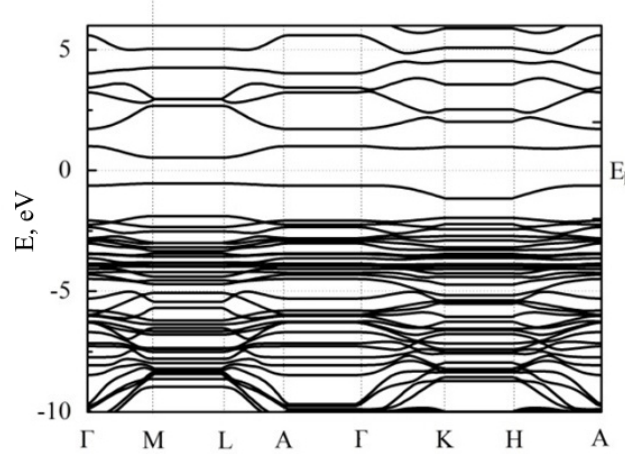

(b)

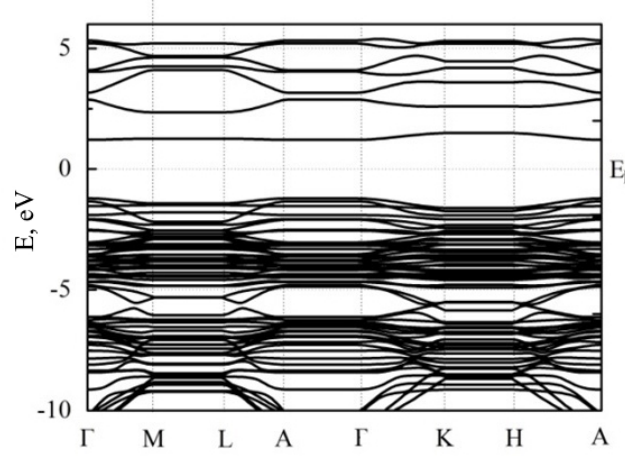

(d)

FIG. 2. The band structure of a graphene layer with chemically adsorbed fluorine atoms on the surface, the number of which per expanded unit cell is: (a) 2 atoms; (b) 6 atoms; (c) 10 atoms; (d) 14 atoms
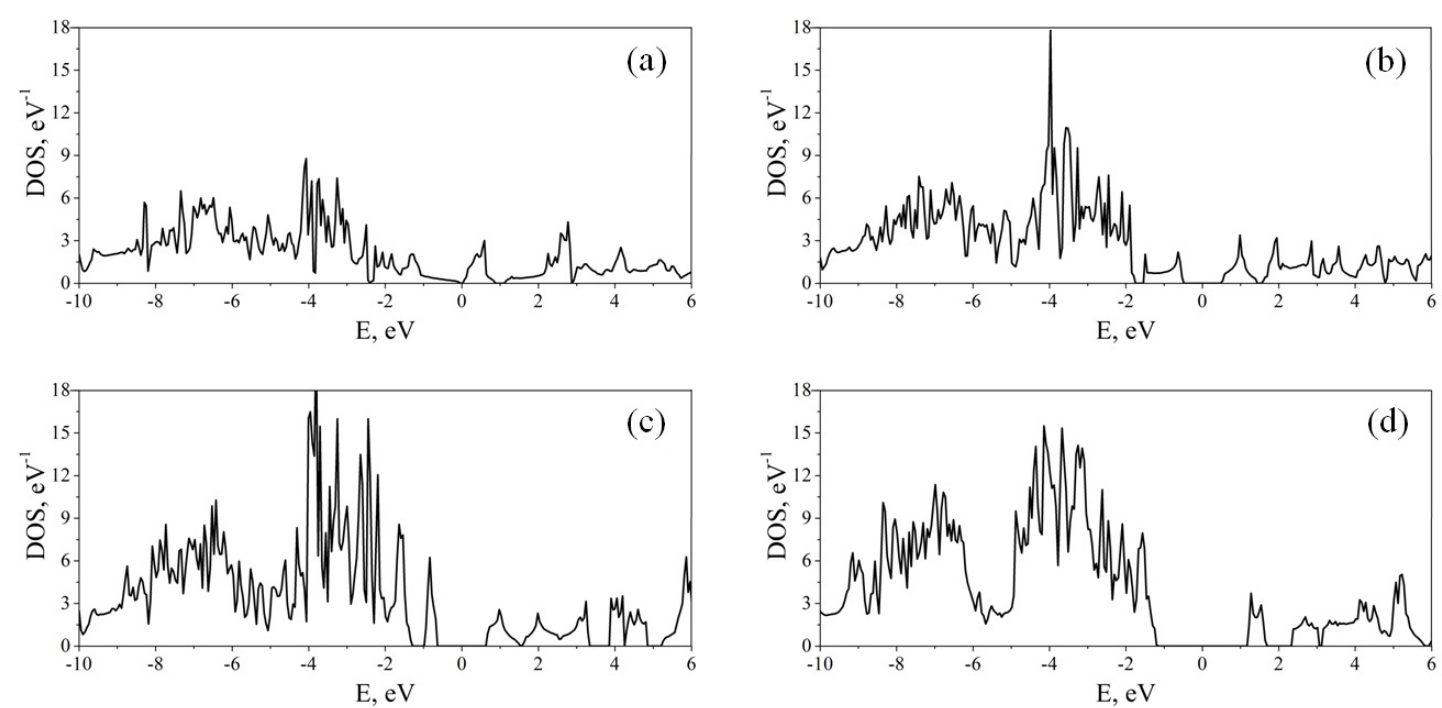

FIG. 3. The density of electronic states (DOS) of a graphene layer with chemically adsorbed fluorine atoms on the surface, the number of which per expanded unit cell is: (a) 2 atoms; (b) 6 atoms; (c) 10 atoms; (d) 14 atoms 


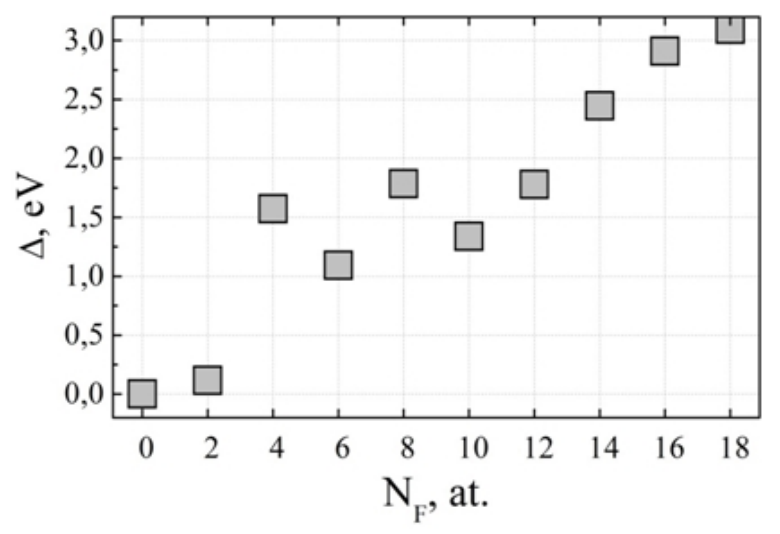

FIG. 4. The dependence of the band gap $(\Delta)$ on the number of fluorine atoms $\left(N_{F}\right)$ which are chemically adsorbed into the unit cell of the graphene layer, which contains 18 carbon atoms

\section{Acknowledgements}

The reported study was funded by Russian Foundation for Basic Research (RFBR), project number 20-32-90002.

\section{References}

[1] Freitag M. Nanoelectronics goes flat out. Nature nanotechnology, 2008, 3,P. 455-457.

[2] Omar G., Salim M.A., et al. Electronic applications of functionalized graphene nanocomposites. In Functionalized graphene nanocomposites and their derivatives. Elsevier, Amsterdam, 2019, P. 245-263.

[3] Sturala J., Luxa J., Pumera M., Sofer Z. Chemistry of graphene derivatives: synthesis, applications and perspectives. Chem. Eur. J., 2018, 24, P. 5992-6006.

[4] Chernozatonskii L.A., Sorokin P.B., Artukh A.A. Novel graphene-based nanostructures: physicochemical properties and applications. Russ. Chem. Rev., 2014, 83(3), P. 251-279.

[5] Robinson J.T., Burgess J.S., et al. Properties of Fluorinated Graphene Films. Nano Lett., 2010, 10 (8), P. 3001-3005.

[6] Nair R.R., Ren W., et al. Fluorographene: a twodimensional counterpart of Teflon. Small, 2010, 6, P. $2877-2884$.

[7] Elias D.C., Nair R.R., et al. Control of graphene's properties by reversible hydrogenation: evidence for graphane. Science, 2009, 323, P. 610613.

[8] Chen D., Feng H., Li J. Graphene oxide: preparation, functionalization, and electrochemical applications. Chem. Rev., 2012, 112 (11), P. 60276053.

[9] Li B., Zhou L., et al. Photochemical chlorination of graphene. ACS Nano, 2011, 5 (7), P. 5957-5961.

[10] Belenkov M.E., Chernov V.M., Belenkov E.A. Structure of fluorographene and its polymorphous varieties. J. Phys.: Conf. Ser., 2018, 1124, 022010 .

[11] Grishakov K.S., Katin K.P., Maslov M.M., Prudkovskiy V.S. Relative stabilities of various fully functionalized graphene polymorphs under mechanical strain and electric field. Applied Surface Science, 2019, 463, P. 1051-1057.

[12] Belenkov M.E., Chernov V.M., Belenkov E.A. Simulation of the structure and electronic properties of fluorographene polymorphs formed on the basis of 4-8 graphene. IOP Conf. Ser.: Mater. Sci. Eng., 2019, 537, 022058.

[13] Belenkov M.E., Chernov V.M. Structure and electronic properties of 4-6-12 graphene layers functionalized by fluorine. Letters on Materials, 2020, 10 (3), P. 254-259.

[14] Withers F., Russo S., Dubois M., Craciun M.F. Tuning the electronic transport properties of graphene through functionalisation with fluorine. Nanoscale Res. Lett., 2011, 6,526.

[15] Yuhua D., Stinespring C.D., Chorpening B. Electronic structures, bonding configurations, and bandgapopening properties of graphene binding with lowconcentration fluorine. Chemistry Open, 2015, 4 (5), P. 642-650.

[16] Koch W.A., Holthausen M.C. Chemists guide to density functional theory. 2nd edition. Wiley-VCH, Weinhein, 2001. 293 p.

[17] Giannozzi P., Baroni S., et al. QUANTUM Espresso: a modular and open-source software project for quantum simulations of materials. Journal of Physics: Condensed Matter, 2009, 21 (39), 395502.

[18] Cheng S.H., Zou K., et al. Reversible fluorination of graphene: Evidence of a two-dimensional wide bandgap semiconductor. Physical Review $B, 2010,81(20), 205435$.

[19] Pierson H.O. Handbook of carbon, graphite, diamond, and fullerenes: properties, processing, and application. Noyes, Park Ridge, New Jersey, 1993, $402 \mathrm{p}$.

[20] Wallace P.R. The band theory of graphite. Physical Review, 1947, 71 (9), P. 622-634.

[21] Novoselov K.S., Geim A.K., et al. Electric field effect in atomically thin carbon films. Science, 2004, 306 (5696), P. 666-669.

[22] Charlier J.C., Gonze X., Michenaud J.P. First-principles study of graphite monofluoride (CF)n. Physical Review B, 1993, 47 (24), P. 1616216168.

[23] Robinson J.T., Burgess J.S., et al. Properties of fluorinated graphene films. Nano Letters, 2010, 10 (8), P. 3001-3005. 